\title{
Potential of Photon Collider in resolving SM-like scenarios
}

\author{
I. F. Ginzburg ${ }^{\mathrm{a}, 1}$ M. Krawczyk ${ }^{\mathrm{b}, 2}$ P. Osland ${ }^{\mathrm{c}, 3}$

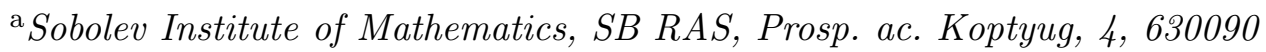 \\ Novosibirsk, Russia \\ ${ }^{\mathrm{b}}$ Institute of Theoretical Physics, Warsaw University, 00-681 Warsaw, Poland \\ ${ }^{\mathrm{c}}$ Department of Physics, University of Bergen, Allegt. 55, N-5007 Bergen, Norway
}

\begin{abstract}
After operations at the LHC and $e^{+} e^{-}$Linear Colliders it may be found that a Standard-Model-like scenario is realized. In this scenario no new particle will be discovered, except a single Higgs boson having partial widths or coupling constants squared with fundamental particles close, within anticipated experimental uncertainty, to those of the SM. Experiments at a Photon Collider can resolve whether the SM model or e.g. the Two Higgs Doublet Model is realized in Nature.

For the SM-like version of the $2 \mathrm{HDM}$ (II) we study the loop couplings of the Higgs boson with $\gamma \gamma$ and $Z \gamma$, and also with gluons. The deviation of the twophoton width from its SM value is generally higher than the expected inaccuracy in the measurement of $\Gamma_{\gamma \gamma}$ at a Photon Collider. The result is sensitive to the parameters of the Higgs self interaction.
\end{abstract}

Key words: Higgs mechanism; Standard Model; Photon Colliders

\section{Introduction}

It could happen that no new particles will be discovered the the Tevatron, the LHC and $e^{+} e^{-}$Linear Colliders [1] except the SM-like Higgs boson. In this case the main task for new colliders will be to search for signals of new physics

1 Supported by RFBR grants 99-02-17211 and 00-15-96691.

2 Partially supported by KBN Grants No 2P03B01414, No 2P03B05119 and the DESY Directoriat.

3 Supported by the Research Council of Norway. 
via deviations of observed quantities from Standard-Model predictions. The study of Higgs boson production at a Photon Collider [2] offers excellent opportunities for this [3]. Indeed, in the SM and in its extensions, all fundamental charged particles contribute to the $h \gamma \gamma$ and $h Z \gamma$ effective couplings. Besides, these couplings are absent in the SM at tree level, appearing only at the loop level. Therefore, the background for signals of new physics will be relatively lower here than in processes which are allowed at tree level of the SM.

In this analysis [4] we assume that an SM-like scenario is realized, i.e., the Higgs particle has been found at the Tevatron or the LHC and its partial widths or coupling constants squared are precisely measured (mainly at the $e^{+} e^{-}$Linear Collider), being close to those of the SM within the anticipated experimental accuracies. This can happen not only in the SM, but also if Nature is described by some other theory, for example, the Two Higgs Doublet Model (2HDM) or the Minimal Supersymmetric Standard Model (MSSM). In the latter cases the observed Higgs boson could be either one of the two neutral scalars. Here we compare the SM and the SM-like scenario in the 2HDM (II).

\section{Standard-Model-like scenario}

The SM-like scenario can be defined by the following criteria:

- One Higgs boson will be discovered with mass above today's limit for an SM Higgs boson [7], $M_{h}>113 \mathrm{GeV}$. This can be either the Higgs boson of the SM or one Higgs boson from the two neutral CP-even scalars $h$ and $H$ $\left(M_{h}<M_{H}\right)$ of the 2HDM or the MSSM.

- The measured decay widths of this Higgs boson (or coupling constants squared) to quarks, charged leptons, EW gauge bosons and gluons, $\Gamma_{i}^{\exp }$ $(i=q, l, W, Z, g)$, will be in agreement with their SM values $\Gamma_{i}^{\mathrm{SM}}$ within the experimental precision

$$
\left|\frac{\Gamma_{i}^{\exp }}{\Gamma_{i}^{\mathrm{SM}}}-1\right| \ll 1 .
$$

- No other Higgs boson will be discovered. Any other Higgs boson is weakly coupled with the $Z$ boson, gluons and quarks, or sufficiently heavy:

$$
M_{H}, \quad M_{A}, \quad M_{H^{ \pm}}>(800 \mathrm{GeV})
$$

to escape observation [8].

- Any other new particle that may exist is beyond the discovery limits of LHC and the $e^{+} e^{-}$Linear Collider. 


\section{Anticipated precision of measured Higgs couplings in the SM- like scenario}

Let $\delta_{i}$ be the relative experimental uncertainty in the partial width, $\Gamma_{i}^{\exp }$ (or coupling constants squared)

$$
\delta_{i}=\frac{\delta \Gamma_{i}^{\exp }}{\Gamma_{i}^{\exp }} .
$$

At the TESLA $e^{+} e^{-}$collider the discussed production cross sections are expected to be measured with a significantly higher precision than at the LHC [8]. At $M_{h} \leq 140 \mathrm{GeV}$ and with integrated luminosity $500-1000 \mathrm{fb}^{-1}$ one can expect [1]:

$$
\begin{aligned}
& \delta_{b}=0.027, \quad \delta_{\tau}=0.062, \quad \delta_{c}=0.137, \quad \delta_{t}=0.055, \\
& \delta_{Z}=0.01, \quad \delta_{W}=0.054, \quad \delta_{g}=0.06, \quad \delta_{\gamma}=0.14 .
\end{aligned}
$$

Experiments at Photon Colliders open new perspectives. In particular, even with a modest integrated luminosity of a $\gamma \gamma$ collider in the high energy peak of about $40 \mathrm{fb}^{-1}$, a $\gamma \gamma$ collider makes it possible to improve on the accuracy in measuring the $h \gamma \gamma$ width up to [9]:

$$
\delta_{\gamma}=0.02 \quad \text { for } M_{h}<140 \mathrm{GeV} .
$$

The accuracy in the measurement of the effective $h Z \gamma(H Z \gamma)$ coupling in the process $e \gamma \rightarrow e h(e \gamma \rightarrow e H)$ is evidently not so high.

We will use the above uncertainties to constrain ratios of actual (in principle measurable) coupling constants of each neutral Higgs scalar $\phi(h$ or $H) 4$ with particle $i$ to the corresponding value for the Higgs boson in the SM,

$$
\chi_{i}^{\phi}=\frac{g_{i}^{\phi}}{g_{i}^{\mathrm{SM}}} .
$$

In the SM-like scenario, for the observed Higgs boson $\phi$ all $\left|\chi_{i}\right|$ are close to 1,

$$
\chi_{i}^{\text {obs }}= \pm\left(1-\epsilon_{i}\right), \quad \text { with }\left|\epsilon_{i}\right| \ll 1 .
$$

The allowed ranges for $\epsilon_{i}$ are constrained by the experimental accuracies $\delta_{i}$, $\left|\epsilon_{i}\right| \leq \delta_{i}$ but there are also additional constraints to these $\epsilon_{i}$ which follow from the structure of the considered model.

$\overline{4}$ Discussing both these scalars, we use the notation $\phi$ for $h$ and $H$. 


\section{Two-Higgs-Doublet Model (II)}

We here consider the CP-conserving Two-Higgs-Doublet Model in its Model II implementation, denoted by 2 HDM (II) $[10,11,5]$. Here, one doublet of fundamental scalar fields couples to $u$-type quarks, the other to $d$-type quarks and charged leptons. The Higgs sector contains three neutral Higgs particles, two CP-even scalars $h$ and $H$, and one CP-odd (pseudoscalar) $A$, and charged Higgs bosons $H^{ \pm}$, it coincides in the 2HDM (II) and in the MSSM.

In the SM-like scenario realized in the 2HDM we need to consider both possibilities: not only the light scalar Higgs boson, $h$, but also the heavier one, $H$, could imitate the SM Higgs boson if the lighter scalar $h$ escapes detection [12], see also [6].

The ratios of the direct coupling constants of the Higgs boson $\phi=h$ to the gauge bosons $V=W$ or $Z$ bosons, to up and down quarks and to charged leptons, relative to their SM values can be expressed via angles $\alpha$ and $\beta[5,10]$ :

$$
\begin{aligned}
& \chi_{V}^{h}=\sin (\beta-\alpha), \\
& \chi_{u}^{h}=\sin (\beta-\alpha)+\cot \beta \cos (\beta-\alpha), \\
& \chi_{d}^{h}=\sin (\beta-\alpha)-\tan \beta \cos (\beta-\alpha),
\end{aligned}
$$

with similar expressions for $\phi=H$. Here $\beta$ parameterizes the ratio of the vacuum expectation values of the two basic Higgs doublets and $\alpha$ parameterizes mixing among the two neutral CP-even Higgs fields. The angle $\beta$ is chosen in the range $(0, \pi / 2)$ and the angle $\alpha$ in the range $(-\pi, 0)$.

The coupling of the charged Higgs boson to the neutral scalars $\phi$ depends on the Higgs-boson masses and on the additional parameter $\lambda_{5}[13]$.

\section{Two-Higgs-Doublet Pattern relation}

The quantities $\chi_{i}^{\phi}$ for the couplings of each scalar (7) (referred to below as basic couplings) are closely related to the observables and in the forthcoming analysis it is more natural to use them, instead of $\alpha$ and $\beta$. Since for each $\phi$ these three $\chi_{i}$ can be expressed in terms of two angles, they fulfill a simple relation (pattern relation), which plays a basic role in our analysis. It has the same form for both $h$ and $H$, namely $\left(\chi_{u}-\chi_{V}\right)\left(\chi_{V}-\chi_{d}\right)+\chi_{V}^{2}=1$, or

$$
\left(\chi_{u}+\chi_{d}\right) \chi_{V}=1+\chi_{u} \chi_{d} .
$$


Furthermore, from Eq. (7) follows an expression for $\tan ^{2} \beta$ :

$$
\tan ^{2} \beta=\frac{\chi_{V}-\chi_{d}}{\chi_{u}-\chi_{V}}=\frac{1-\chi_{d}^{2}}{\chi_{u}^{2}-1} .
$$

In the following discussion we will assume only one value for each up-type quark, down-type quark, charged lepton and gauge boson coupling with the Higgs boson, in numerical calculation we will use the best estimate for each category, e.g. $\delta_{b}$ for $\delta_{d}$, etc.

\section{Allowed ranges for couplings}

The SM-like scenario means, in particular, that $\chi_{i}^{2} \approx 1$ (here, we consider only basic couplings with $i=u, d, V$; loop couplings are discussed in the next section). We consider solutions of the equations (6) constrained by the pattern relation (8). Taking into account the definitions (7) and earlier noted regions of variation of the mixing angles $\alpha$ and $\beta$, we check if the obtained solution can be classified as SM-like with respect to the first criterion of Sec. 2. For the observed Higgs boson $\phi$ we consider solutions denoted $A_{\phi 1}$ and $A_{\phi 2}$ with approximately identical $\chi_{V} \approx \chi_{u} \approx \chi_{d} \approx \pm 1$. Subscript 1 corresponds to solutions with $\chi_{V} \approx 1$ while subscript 2 labels solutions with $\chi_{V} \approx-1$. These solutions are really close to the SM for all basic couplings: relative phases coincide, and magnitudes are practically the same. There are also solutions where some of the $\chi_{i} \approx 1$ but other $\chi_{j} \approx-1$. These are denoted $B_{\phi 1}$ and $B_{\phi 2}$, where the subscripts have the same meaning as above. These solutions are in fact distinct from the SM case, even though all basic widths $\sim \chi_{i}^{2}$ are close to the SM values; we will not discuss them here, for details see [4].

For the solutions $A_{H i}$ where the observed SM-like Higgs boson is the heavier one, the values for the basic couplings can describe a picture which is different from the SM-like scenario since the lighter Higgs boson can in principle be observable. We discuss these possibilities in each case and exclude some solutions from the subsequent discussion, if they do not fulfill the criteria of Sec. 2 (no other Higgs particle should be discovered).

For the solutions A near the SM point for all basic coupling constants: $\chi_{V}=$ $\chi_{u}=\chi_{d}= \pm 1$, i.e.,

$$
\begin{gathered}
\chi_{V}=1-\epsilon_{V}, \quad \chi_{d}=1-\epsilon_{d}, \quad \chi_{u}=1-\epsilon_{u}, \quad\left(A_{h 1}, A_{H 1}\right) \\
\chi_{V}=-1+\epsilon_{V}, \quad \chi_{d}=-1+\epsilon_{d}, \quad \chi_{u}=-1+\epsilon_{u} .\left(A_{h 2}, A_{H 2}\right),
\end{gathered}
$$

we obtain, using the pattern relation and neglecting terms of higher order in 
$\epsilon_{i}$

$$
\tan \beta=\frac{\left|\epsilon_{d}\right|}{\sqrt{2 \epsilon_{V}}}=\sqrt{\left|\frac{\epsilon_{d}}{\epsilon_{u}}\right|}, \quad \epsilon_{u}=-\frac{2 \epsilon_{V}}{\epsilon_{d}} \Rightarrow \epsilon_{V}=-\frac{\epsilon_{u} \epsilon_{d}}{2}<\frac{\delta_{u} \delta_{d}}{2} .
$$

According to Eqs. (6) and (7), $\epsilon_{V}>0$, so that in all these solutions the signs of $\epsilon_{u}$ and $\epsilon_{d}$ are opposite. Since $\epsilon_{V}$ here is given by the product of two other $\epsilon$ 's, it should be extremely small, $\left(\epsilon_{V} \leq 0.001\right.$ using $\delta_{t}$ and $\delta_{b}$ from Eq. (4), while for $V=Z$ we expect $\left.\delta_{Z}=0.01\right)$. This deviation can therefore be neglected, and in the calculations of loop-induced couplings one can put $\chi_{V}= \pm 1$.

\section{Distinguishing models via loop couplings}

In order to distinguish models in the considered SM-like scenario, we compute loop-induced couplings of the Higgs boson with photons or gluons $[10,13]$ for solutions $A_{h}$ and $A_{H}$ within the ranges of the coupling constants allowed by the anticipated experimental inaccuracies from Eq. (4) and within the constraints of the pattern relation, Eqs. (10) and (11). To estimate the deviation from the SM, we consider the ratios of widths $\chi_{\gamma \gamma}^{2}$ and $\chi_{Z \gamma}^{2}$ obtained in the 2HDM (II) and in the SM. In the 2HDM the couplings with photons, $h \gamma \gamma$ and $h Z \gamma$, contain contributions from fermions, and from the charged gauge boson $W^{ \pm}$, like in the SM. In addition, there are contributions from the charged Higgs boson, $H^{ \pm}$.

For definiteness, we perform all calculations here for $M_{H^{ \pm}}=800 \mathrm{GeV}$. At $M_{\phi}<250 \mathrm{GeV}$ the contribution of the charged Higgs boson loop varies by less than $5 \%$ when $M_{H^{ \pm}}$varies from $800 \mathrm{GeV}$ to infinity.

The $\gamma \gamma$ and $Z \gamma$ widths look the most promising ones for distinguishing models. A new feature of these widths, as compared to the SM case, is the contribution due to the charged Higgs boson loops. It is known that the scalar loop contribution to the photonic widths is less than that of fermion and $W$ boson loops (the last is the largest). The contributions of $W$ and $t$-quark loops are of opposite sign, i.e., they partially compensate each other, thus, the effect of scalar loops is enhanced here. The coupling $\chi_{H^{ \pm}}$depends on $\lambda_{5}$, which is not fixed by the observable masses. This dependence is linear in the considered partial widths

$$
\chi_{a}^{2}=\frac{\Gamma_{a}^{2 \mathrm{HDM}}}{\Gamma_{a}^{\mathrm{SM}}}=1-R_{a}(1-\Lambda), \quad a=\gamma \gamma \text { or } Z \gamma,
$$

with $\Lambda$ proportinal to $\lambda_{5}$. 
For $\lambda_{5}=0$ the ratios of the considered Higgs widths to their SM values are shown in Fig. 1. Here, thick curves correspond to strict SM values for the basic couplings with quarks and gauge bosons. These curves are below unity due to the contribution of the charged Higgs boson. The shaded regions are derived from the anticipated $1 \sigma$ bounds around the SM values of two measured basic coupling constants, $g_{b}$ and $g_{t}$ (the third basic coupling $g_{Z}$ must be very close to its SM value). Solutions A cover the shaded area in Fig. 1. With increasing Higgs boson mass, the deviation of the considered loop couplings from their SM values decreases monotonically.
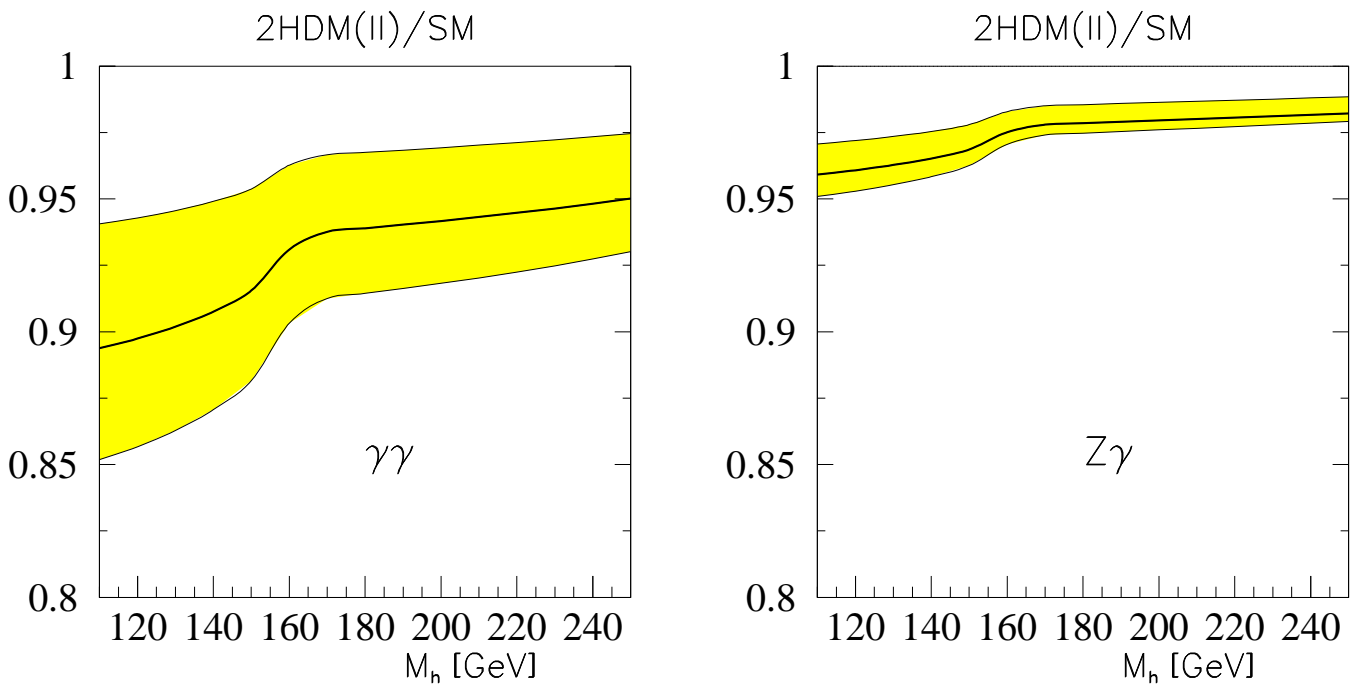

Fig. 1. Ratios of the Higgs boson $\phi \rightarrow \gamma \gamma$ and $\phi \rightarrow Z \gamma$ decay widths in the 2HDM and the SM as functions of $M_{h}$ for all solutions $A$ with $\lambda_{5}=0$. See text for description of the shaded bands.

We see that the deviation from unity is large enough to allow a reliable distinction of the 2HDM from the SM in the process $\gamma \gamma \rightarrow H$. This conclusion is valid in a wide range of $\lambda_{5}$ values. The possible precision in the determination of $\lambda_{5}$ from the two-photon width depends crucially on the mass of the charged Higgs boson.

The two-gluon width is determined by the contributions of $t$ and $b$ quarks. For not too high values of $\tan \beta$, the $t$-quark contribution dominates. So, the difference $\chi_{g g}-1$ is determined by the difference $\chi_{u}-1$, and with high accuracy $\chi_{g g}-1 \approx 2\left(\chi_{u}-1\right)$. If $\tan \beta \ll 1$ then the deviation of the Higgs boson coupling with $t$-quark from its SM value can be large compared to expected experimental uncertainty (4). In this case the two-gluon width can differ from its SM value by more than the experimental uncertainty, and the measurement of the two-gluon width could exclude the SM-like scenario from being realized by the 2HDM. In such a case the Photon Collider can be used for a more detailed study of the realized model beyond the SM. 


\section{Conclusion}

An SM-like scenario observed at the LHC and $e^{+} e^{-}$Linear Colliders can occur both in the SM and in other models, including the 2HDM (II). In order to distinguish these models, we implement a pattern relation among basic couplings. Taking into account anticipated uncertainties in future measurements of the basic couplings of the Higgs boson, we found that the pattern relation in the considered SM-like scenario, in which partial widths of Higgs boson decay are close to their SM values, has two types of solutions. In the solutions $A$ all basic couplings are close to their SM values. (In the solutions $B$, some of the basic couplings are close to their SM values while others differ in sign from the SM values.)

We studied the $\gamma \gamma$ and $Z \gamma$ partial widths of the observed Higgs boson for all considered solutions. The obtained predictions are practically identical for the cases when the observed scalar is the lighter Higgs boson of 2HDM $(h)$ or the heavier one $(H)$. For solutions $A$ this difference depends on an additional parameter of the theory, $\lambda_{5}$. These solutions can be discriminated in a considerable range of values of this parameter. The $\gamma \gamma$ width in the 2 HDM (II) differs significantly from the corresponding SM width in a wide range of parameters. Therefore, with the anticipated high accuracy of measuring this width at a Photon Collider, such a measurement could in general resolve the 2HDM (II) and the SM.

\section{References}

[1] E. Accomando et al., Phys. Rept. 299, 1 (1998);

See also TESLA Technical Design Report, to be published. Previous estimates can be found in M. Battaglia, Proc. 4th Int. Workshop on Linear Colliders, April 28-May 5, 1999; Sitges (Spain), p. 163 (hep-ph/9910271); A. Djouadi, ibid., p. 51 (hep-ph/9910449); P. Zerwas, hep-ph/0003221.

[2] I.F. Ginzburg, G.L. Kotkin, V.G. Serbo, V.I. Telnov, Nucl. Instrum. Methods 205, 47 (1983); I.F. Ginzburg, G.L. Kotkin, S.L. Panfil, V.G. Serbo, V.I. Telnov, Nucl. Instrum. Methods, A 219, 5 (1984); Zeroth-order Design Report for the $N L C$, SLAC Report 474 (1996); R. Brinkmann et al., Nucl. Instrum. Methods, A 406, 13 (1998).

[3] I. Ginzburg, M. Krawczyk, P. Osland, Proc. 4th Int. Workshop on Linear Colliders, April 28-May 5, 1999; Sitges (Spain) p. 524; LC note LC-TH-2000039 (hep-ph/9909455).

[4] I. Ginzburg, M. Krawczyk, P. Osland, IFT 2000-21, hep-ph/0101208, to be published. 
[5] H.E. Haber, Talk given at Beyond the Standard Model Conf., Lake Tahoe, CA, Dec. 13-18, 1994 and at Ringsberg Workshop on Perspectives for Electroweak Interactions in $e^{+} e^{-}$Collisions, Ringsberg, Germany, 5-8 Feb. 1995, hepph/9505240.

[6] B. Grządkowski, J.F. Gunion, J. Kalinowski, Phys. Rev. D 60, 075011 (1999); Phys. Lett. B 480, 287 (2000).

[7] C. Tully, Status of LEP-wide Higgs searches, 5 September 2000 (LEP Higgs working group).

[8] See e.g., A. Djouadi et al. (Higgs working group), Workshop on Physics at TeV Colliders, Les Houches, France, June 1999, hep-ph/0002258;

ATLAS detector and Physical Performance Technical Design Report, http://atlasinfo.cern.ch/Atlas/GROUPS/PHYSICS/TDR/access.html, G. L. Bayatian et al., CMS Technical Proposal, CERN LHCC 94-38 (1994), http://cmsinfo.cern.ch/TP/TP.htm]; D. Zeppenfeld, hep-ph/0005151.

[9] M. Melles, W.J. Stirling, V.A. Khoze, Phys. Rev. D 61, 054015 (2000); G. Jikia, S. Söldner-Rembold, Nucl. Phys. B (Proc. Suppl.) 82, 373 (2000).

[10] J.F. Gunion, H.E. Haber, G. Kane, S. Dawson, The Higgs Hunter's Guide (Addison-Wesley, Reading, 1990).

[11] R. Santos and A. Barroso, Phys. Rev. D 56, 5366 (1997).

[12] M. Krawczyk, in Proceedings of the 28th International Conference on High Energy Physics, ICHEP '96, editors Z. Ajduk and A.K. Wroblewski (World Scientific, Singapore, 1997) p. 1460; M. Krawczyk and J. Żochowski, Phys. Rev. D 55, 6968 (1997); M, Krawczyk, J. Żochowski, P. Mattig, Eur. Phys. J. C 8, 495 (1999); P.H. Chankowski, M. Krawczyk, J. Żochowski, Eur. Phys. J. C 11, 661 (1999);

M. Krawczyk, P. Mattig, J. Żochowski, Eur. Phys. J. C in press, hepph/0009201;

P. Chankowski et al., hep-ph/0009271, Phys. Lett. B, to appear.

[13] A. Djouadi, V. Driesen, W. Hollik, A. Kraft, Eur. Phys. J. C 1, 163 (1998). 